Jean-Marie SECA, « Formatage du ressentir et représentations underground », Sociétés, vol. 90, $\mathbf{n}^{\circ} 4$, octobredécembre, 2005, pp. 57-69.

\title{
Formatage du ressentir et représentations underground
}

Par Jean-Marie SECA

Mots-clés : sensibilité, mutation, représentations musicales, standardisation

Key words: feeling, transformation, musical representations, standardization

\section{Résumé}

Au fur et à mesure de la succession des générations jeunes, depuis une soixantaine d'années, on note l'émergence progressive d'une naturalisation des pratiques de musique amplifiées (rock, metal, techno, rap). Ce contexte toujours plus fortement dominant est caractérisé par la multiplication d'expériences, tant créatives que consommatrices, de ces styles. La techno et ses variantes plus ou moins subversives ou ludiques en sont à la fois les derniers avatars et les espaces-temps d'intensification tant par les rythmes saccadés ou ultrarapides que dans la valorisation des états modifiés de conscience. Après en avoir décrit les grandes caractéristiques, on développera la thèse de l'existence d'injonctions paradoxales dans les emprises soniques suscitées. Les courants underground, même fragmentés et groupusculaires, seraient-ils parvenus à une situation de massification en devenant un savoir partagé par un nombre de plus en plus grand d'adeptes?

\section{Abstract}

Progressively with the succession of the young generations, since an about sixty years, one notes the progressive emergence of a naturalization of the amplified practices of music (rock'n'roll, metal, techno, rap). This context always more strongly dominating is characterized by the multiplication of experiments, as well creative as consuming, of these styles. The techno and its alternatives more or less subversive or ludic are at the same time the last changes and the space times of intensification as well by the jerked or high-speed rhythms as in the valorization of the modified states of consciousness. After having described the great characteristics of them, one will develop the thesis of the existence of paradoxical injunctions in the caused sonic influences. Would these underground styles, even fragmented and groupuscule, have arrived at a situation of massification while becoming a knowledge shared by an increasingly large number of followers? 
Jean-Marie SECA, « Formatage du ressentir et représentations underground », Sociétés, vol. 90, $\mathbf{n}^{\circ}$ 4, octobredécembre, 2005, pp. 57-69.

\section{Introduction}

Sans être vraiment disposé à cela, je vais tenter, dans cet article, de grossir le trait des reproches adressés aux musiques actuelles. Je formulerai parallèlement des commentaires sur leur nature psychosociale et leur force d'emprise. S'il y a « du politique » dans la techno, il ne correspond pas à une vision machiavélique ou verticale. II s'agit plutôt d'un sens horizontal, aristotélicien, renvoyant à un ethos et à des figures de style. S'il pense à la première connotation, Michel Maffesoli a bien sûr raison de dire que la techno et ses variantes ne sont pas politiques ${ }^{1}$. La seconde signification (horizontale, éthique, dialogique) est plus dérangeante pour les élites. Alexandre Dorna y voit une proposition alternative souvent négligée par les institutions et les gouvernants. II s'agit de «l'existence d'un dispositif délibératif capable de rendre viable la multiplicité des prises de parole et l'interdiction de l'emprise d'un seul discours. » La politique, dans cette conception-là, est donc «l'ensemble des discours dont l'horizon n'est pas [l'obtention d'un] plus de pouvoir mais le bien vivre $»^{2}$. II faut insister sur un point avant d'avancer dans notre propos. La techno, a renoué avec une conception romantique des subcultures. Elle a cantonné la dimension politique à des combats plus ou moins ludiques, juridiques, paramilitaires ou à des actions de lobbying sur les décideurs. On s'y affirme contre les interdits et les autorités de l'État tout autant que pour la festivité projetée. Cette musique n'est pas la seule à exprimer cette rhétorique de catégorisation sociale du « seul contre le pouvoir » et de «la résistance contre la majorité bien-pensante ». C'est pourquoi on peut y détecter une certaine stéréotypie et une répétitivité étonnante. II faut cependant penser les micro-mouvements musicaux comme des « sémiotisations » de la révolte et de la protestation. II faut seulement comprendre ce que ces rébellions contiennent quand elles se transfigurent en machineries musicales. C'est ce que j'essaierai de faire partiellement dans la suite de ce texte. Dans un premier temps, je propose de coincer ces musiques populaires amplifiées ainsi que la techno entre le marteau de l'idéologie et l'enclume de l'utopie. Les débats de ce genre ont du bon dans le seul but de sortir du sentier des discours partisans, pourvu qu'ils se limitent à un paragraphe. Je décrirai ensuite ce que j'estime être leur structure de socialisation (dans le faire, les représentations, le style ou les objectifs de production). Dans un troisième moment, je me focaliserai sur les enjeux de pouvoir sur la masse (emprise sonique), de besoin de reconnaissance sociale et de transe. Pour conclure, je tenterai de critiquer ces courants qu'on a trop l'habitude d'encenser sous prétexte qu'ils ne sont pas académiques et qu'ils représenteraient des cultures d'outsiders 3 . Le commentaire que je formulerai porte sur que je qualifie de

\footnotetext{
${ }^{1}$ MAFFESOLI M., « Une démesure sage et nécessaire » (entretien avec B. Mabillon-Bonfils), in MABILLON-BONFILS B. (éd.), La fête techno. Tout seul et tous ensemble, Paris, Autrement, 2004, pp. 68-69.

2 DORNA A., De l'âme et de la cité. Crise, populisme, charisme et machiavélisme, Paris, L'Harmattan, 2004. pp. 83-84.

${ }^{3}$ BECKER H. S., Outsiders. Études de sociologie de la déviance, Paris, Métailié, 1985 (1re éd. en langue anglaise : 1963).
} 
Jean-Marie SECA, « Formatage du ressentir et représentations underground », Sociétés, vol. 90, n 4, octobredécembre, 2005, pp. 57-69.

« musique underground de masse » et de «standardisation du ressentir ». Je considère, peut-être à tort, qu'il faut savoir en décrypter la symptomatologie. Tentons, dans la suite de ce texte, de proposer quelques arborescences interprétatives dont l'inspiration n'est pas du tout fondée sur du mépris mais plutôt sur le regard distancié de l'ethnographe qui se permet ce repli dans la froide raison après diverses enquêtes de terrain 4 .

\section{Entre le marteau idéologique et l'enclume utopique?}

Certaines des réflexions suivantes recèlent un air de déjà-vu. Le débat ${ }^{5}$ a été initié dans les années 1930 et, même avant, si l'on se réfère à la genèse de la culture de masse ${ }^{6}$. II faut cependant pouvoir poser le problème de la reproduction et de la transmission d'un mode d'être dans les musiques actuelles. On observe, en effet, l'ancrage de systèmes de croyances et de représentations, relativement récurrents, tendant vers une formalité discursive, de type assertif, évaluatif, convenu, voire convenable, portant notamment et pour ce qui concerne le propos de cet article, sur les objets « musique », « art », « fête », « drogue », « transe » ou « apprentissage technique ». Ces cultures font aussi référence à des valeurs, à des types d'émotions et à divers conformismes majoritaires (culte de la vie d'artiste, valorisation du festoiement, esthétismes à la mode) diffusés par les médias. Elles peuvent conduire à une aliénation et à une déprivation sensorielle, isolant celui qui s'y connecte, de certaines potentialités, ressources ou représentations effectivement alternatives, c'est-à-dire proposant une bifurcation vers des sensibilités divergentes. Elles iraient donc de pair avec une forme d'enfermement cognitif, - comme tout produit artistique me dira-t-on -, et seraient accompagnées de conduites anomiques. Au sens propre du terme, elles isolent, tout en reliant à un autrui vaporeux, électro-amplifié, transporté par un état de transe plus ou moins aidé chimiquement. Cette transe, tant recherchée, peut d'ailleurs résulter d'autres facteurs aussi bien personnels qu'extramusicaux comme, par exemple, un effet de relaxation résultant d'un contraste radical entre le monde festif et celui (du travail ou des études) causant (de plus en plus) le stress.

Au-delà des recherches sonores et des bricolages musicaux reprenant une façon de faire contemporaine, l'effervescence expressive des pop cultures illustre une représentation en vogue du plaisir, du charisme et de l'émotion. Le feeling éprouvé condense un esprit du temps tendanciellement

\footnotetext{
4 SECA J.-M., Les musiciens underground, Paris, PUF, 2001 (traduction en castillan : 2004).

${ }^{5}$ ADORNO T., Le caractère fétiche dans la musique et la régression de l'écoute, Paris, Allia 2001 (1re éd. : 1938).

6 LOHISSE J., Les systèmes de communication. Approche socio-anthropologique, Paris, A. Colin, 1998.

GUIBERT G., Scènes locales, scène globale. Contribution à une sociologie économique des producteurs de musiques amplifiées en France, Thèse pour le doctorat de sociologie, Nantes, Université de Nantes, 2004.
} 
Jean-Marie SECA, « Formatage du ressentir et représentations underground », Sociétés, vol. 90, n 4, octobredécembre, 2005, pp. 57-69.

présent dans des spectacles « grand public » ou narré dans les récits épiques sur les héros éponymes de chaque «mini-tribu » stylistique. II ne s'agit évidemment pas de quelque chose d'imposé. Les musiques actuelles ou moins récentes ne délivrent pas de doctrine attestée, comme le marxisme et ses chapelles théoriques ou militantes. Une tendance normalisatrice y prévaut néanmoins. Certains apologues appartenant au milieu journalistique spécialisé ou issus parfois de la recherche l'énoncent plus ou moins clairement au nom d'une approche compréhensive et glissent imperceptiblement vers la fascination ; divers adeptes reprennent, malgré tout, dans leur fougueuse militance spontanée, à tuetête et bras-le-corps, des paroles de « morceaux » et / ou certains rythmes. Si l'on pouvait décrire un « engagement » dans ces pratiques, on devrait parler d' « actes militants pour rire », ne durant que le temps d'une soirée, pour les consommateurs, ou de quelques années, pour les producteurs et autres Sound Systems ${ }^{7}$. Précisons, en passant, qu'en dépit de cette gratuité relative et de cette légèreté nécessaire, les disputes entre esthètes peuvent engendrer de vraies batailles comme par le passé. ॥ suffit de rappeler la querelle d'Hernani, en 1830, ou celle des Bouffons, à partir de 1752. Les conflits sur la beauté et l'art sont aussi des affaires sérieuses malgré l'affectation d'une insouciance par certains. De plus, la distraction prend beaucoup de temps et d'énergie sans parler de l'implication des artisans du son qui investissent énormément dans leur passion8.

Un système de représentations organise l'influence des productions électroniques. II est constitué tout autant de discours (intellectualisés quand les performances ne recèlent pas de paroles), d'émotions, d'actes que de symboles et de cristallisations esthétiques. Il renvoie à une série articulée d'évidences et d'affirmations sur la rébellion, l'être ensemble, le corps ou le plaisir. II objective une vision du monde, qualifiée d' «utopie » par ses supporters ou ses intellectuels organiques et d' «idéologie » par ses adversaires ou ses analystes distanciés. Ceux qui perçoivent une utopie dans la techno, le rap ou le rock n'acceptent pas de considérer ces genres comme une tradition rituelle des jeunes de notre époque. Ces derniers seraient ainsi en recherche d'un « liant » psychosocial relevé, en symbiose avec un groupe d'appartenance idéalisé. Ils seraient obnubilés par une norme d'originalité exacerbée en vue de fixer une identité flottante sur des espaces prothétiques malléables. En détournant une expression inventée par Serge Moscovici, ces cultures esthétiques renverraient à des tendances proches des « religions profanes $»^{9}$. Celles-ci seraient, en dernier ressort, des visions du monde rassurantes, axées sur l'amour de la masse vis-à-vis de ses propres manifestations et oeuvres. Ces cultures musicales ont transfiguré l'implication dans les mouvements politiques auxquels pensait Moscovici quand il établissait

7 POURTAU, L., « Les Sound Systems technoïdes, une expérience de la vie en communauté, in MABILLON-BONFILS B. (éd.), La fête techno. Tout seul et tous ensemble, Paris, Autrement, 2004, 100-114.

8 POURTAU, op. cit.

${ }^{9}$ MOSCOVICI S., L'âge des foules. Un traité historique de psychologie des masses, Paris, Fayard, 1981. 
le contenu de la première fonction d'une religion profane : « composer une vision du monde, qui pallie le caractère fragmentaire et divisé de chaque science, de chaque technique et de la connaissance en général. »10. Sa seconde utilité « consiste à harmoniser les rapports entre l'individu et la société, à réconcilier en lui les tendances sociales et antisociales. »11. La troisième est de dissimuler un mystère. «Le secret serait celui de la croyance pour l'amour de laquelle les hommes endurent les pires supplices », chacun renonçant à la vérité pour rester dans la communauté12. Les styles underground sont évidemment loin d'être des églises. Ils constituent cependant des cultures sectaires quasireligieuses et voisinent parfois avec le mysticisme comme, par exemple, dans le metal13.

Ceux qui n'y lisent qu'une reproduction d'un modèle commercial sophistiqué ne veulent pas comprendre que, dans les masses, surgissent, malgré tout, des minorités virulentes formées de leaders qui tracent, avec rage et intelligence, des matrices harmoniques, rythmiques, poétiques et des revendications plus ou moins explicitées, proposant un autre scénario pour le vivre-ensemble. Dans ce dernier cas, on peut parler d'activismes esthétiques erratiques. La flamme des conduites de transformation culturelles est transmise, de façon privée, clandestine, entre les diverses générations jeunes composant involontairement des alliances stylistiques variées, bruyantes et «corporéisées » depuis une soixantaine d'années. Dans mes écrits, je parle de «mutation sensible » au long cours. Cette émergence dynamique et artistique fait, en partie, penser aux tableaux du peuple en marche chantant la Marseillaise ou bien à ceux de partisans combattant pour la liberté. II y a une geste pure et une force dialectique du groupe charismatique à l'état naissant dans ces mouvances. Le malentendu entre les premiers (apologues) et les seconds (intellectuels critiques ou / et réactionnaires au sens propre du terme) est remarquable. II ne finira pas de l'être.

La thèse de l'opium des masses peut-elle être envisagée ? Karl Mannheim écrivait sentencieusement, il y a plus de trois-quarts de siècle : « Nous voici [...] en face de cette tendance étrange et effrayante de la pensée moderne, dans laquelle l'absolu qui était autrefois un moyen d'entrer en communication avec le divin, est maintenant devenu un instrument employé par ceux qui en tirent profit pour déformer,

\footnotetext{
$10 \mathrm{MOSCOVICl}$, op. cit., p. 462.

11 Ibid., p. 464.

12 Ibid., p. 467 et 470.

13 HEIN F., Hard rock, heavy metal, metal... Histoire, cultures et pratiquants, Clermont-Ferrand, Mélanie Séteun, 2004.

MOMBELET A., La religion metal. Secte metal et religion postmoderne, Mémoire pour le DEA de sociologie, Paris, Université de Paris-5, 2004.
}

WALZER N., L'imaginaire satanique et néo-païen dans la musique metal extrême depuis les années 1990 (à travers la perception française), Mémoire pour la maîtrise d'histoire, Marne-la-Vallée, Université de Marne-la-Vallée, 2003. 
Jean-Marie SECA, « Formatage du ressentir et représentations underground », Sociétés, vol. 90, $\mathbf{n}^{\circ}$ 4, octobredécembre, 2005, pp. 57-69.

pervertir et dissimuler la signification du présent ${ }^{14}$. Le sociologue, en parlant des «déformations idéologiques », les décrit comme des cas de «fausses consciences » ou de ce que, dans d'autres approches, on pourrait nommer «névroses ». Elles nous conduiraient à essayer de «résoudre des conflits et des situations d'anxiété en recourant à des absolus, alors qu'il n'est plus possible de vivre en harmonie avec ceux-ci. C'est le cas lorsque nous créons des "mythes", que nous adorons "la grandeur en elle-même", lorsque nous déclarons notre soumission à des "idéaux" tandis que, dans notre conduite réelle, nous obéissons à d'autres intérêts que nous essayons de masquer en simulant une rectitude inconsciente, qui n'est que trop facilement transparente $»^{15}$. Les utopies sont, dans la préface à cet ouvrage du sociologue allemand, définies par Louis Wirth, comme « ces complexes qui tendent à créer des activités en vue d'un changement dans l'ordre dominant ». Mannheim ne considère pas l'utopie comme fondamentalement différente, en tant qu'objet étranger à la réalité et élaboration symbolique désynchronisée, de l'idéologie. Ce qui caractérise la première notion est le fait qu'elle tente de dépasser l'existence réelle, de favoriser le passage à l'action et d'ébranler, partiellement ou totalement, l'ordre des choses ${ }^{16}$. Entre la lumière obscure et crue de l'idéologie et la machine à prédire un futur qui chante, les productions de musiques actuelles sculptent un présent qui dure.

\section{Une manière commune et ancienne de faire de la musique}

L'idée que les courants rocks peuvent être présentés comme des matrices des tendances techno est loin d'être inappropriée : Bertrand Voisin et moi-même ${ }^{17}$ sommes allés à contre-courant d'une volonté de voir dans ces derniers styles des événements sonores inouïs et des pratiques absolument originales ${ }^{18}$. Ces tendances électroniques «musiquent » effectivement leur époque. Mais si elles la représentent, c'est aussi parce que le marketing s'en empare et les diffuse globalement sous une forme édulcorée. Leurs développements les plus exemplaires peuvent être analysés comme des intensifications et des extensions de l'état d'esprit animant les courants antérieurs ${ }^{19}$. Si on prend la

\footnotetext{
${ }^{14}$ MANNHEIM K., Idéologie et utopie. Une introduction à la sociologie de la connaissance, Chicoutimi, Uqam (édition électronique : http://www.uqac.uquebec.ca/), 1929 (116 p.), p. 47.

15 MANNHEIM, op. cit., p. 54.

16 Ibid., p. 63.

17 SECA J.-M. et VOISIN B., « Éléments pour une appréhension structurale et socio-historique de la représentation sociale de la musique dans les courants techno et punk », in Copyright Volume!, vol. 3, n 1, 2004, 73-89.
}

18 GAILLOT M., Sens multiple. La techno : un laboratoire artistique et politique du temps présent, Paris, Dis Voir, 1999. GRYNSZPAN E., Bruyante techno. Réflexion sur le son de la free party, Nantes, Mélanie Séteun, 1999.MABILLONBONFILS B. (éd.), La fête techno. Tout seul et tous ensemble, Paris, Autrement, 2004.

RACINE É., Le phénomène techno. Clubs, raves et free-parties, Paris, Imago, 2002.

${ }^{19}$ CASTANET P.-A., Tout est bruit pour qui a peur. Pour une histoire sociale du son sale, Paris, TUM, Michel de Maule, 1999. 
Jean-Marie SECA, « Formatage du ressentir et représentations underground », Sociétés, vol. 90, $\mathbf{n}^{\circ}$ 4, octobredécembre, 2005, pp. 57-69.

peine de se pencher sur les déclarations de diverses figures des courants du passé, on note, par exemple, que le refus puritain de l'idolâtrie y est une préoccupation centrale. La machine commerciale rock se constitue, par ailleurs, dès le départ, comme une usine à intégrer des inconnus plus ou moins doués des cités et des foules, accélérant ce mouvement perpétuel, de type « jeuniste », d'appropriation d'un nouveau courant au fur et à mesure de la succession des générations ${ }^{20}$. La valorisation de l'anonymat de l'artiste techno n'est pas exceptionnelle: dans les groupes punks, une violence mimétique, entre les représentants et les « représentés », était fortement ritualisée. II en est de même pour la transformation des moyens de produire le son et la miniaturisation qui, au début des années 1950, ont pu être pensés comme une régression²1 par rapport aux big bands de jazz des années 1930 et 1940. Le trio « chant - basse - batterie » était, en effet, une aberration identique à la singularisation à l'unique lecteur platine branché à divers appareillages informatiques dans les formes plus actuelles.

Toute une réappropriation des friches industrielles et des environnements urbains a été initiée par des punks et des postpunks ${ }^{22}$ précurseurs des travellers ${ }^{23}$. Une perspective socio-historique permet de délimiter une série d'emboîtements et de déterminations successives dans l'intériorisation de ces musiques, d'emprunts culturels, techniques et d'approfondissement de logiques commerciales, créatives ou artistiques qui étaient précédemment en germes et plus éparses. Le collage et l'altération de versions d'un standard est, depuis des dizaines d'années, une tradition du blues et du jazz. Les remix triturés de morceaux « classiques » rock ou électroniques en sont une continuation. Un groupe américain, The Residents, rarement cité par les historiens de la genèse de la techno, n'hésitait pas, durant les années 1970, à proposer un album cynique et typiquement adapté à l'esprit punk de l'époque, The Third Reich of Rock'n Roll. II reprenait, en 1989, de façon similaire à ce qui se fait aujourd'hui et depuis dix ans, des morceaux connus, comme les grands standards d'Elvis Presley, en les remodelant à leur manière. L'histoire des styles populaires ou non fourmille de ce genre d'exemples ${ }^{24}$. Et quand on parle des followings ou ensembles de producteurs, supporters, poètes et

\footnotetext{
20 BUXTON D., Le rock. Star système et société de consommation, Grenoble, La pensée sauvage, 1985.

21 BUXTON, op. cit.

22 FOURNIER V., Les nouvelles tribus urbaines. Voyage au cœur de quelques formes contemporaines de marginalité culturelle, Chênes-Bourg, Georg, 1999.
}

VOISIN B., La géographie du mouvement punk, mémoire pour la maîtrise d'aménagement du territoire, Paris, Université de Paris IV-Sorbonne, 2002.

${ }^{23}$ DELORME A., « Le processus d'individualisation en situation de précarité : deux communautés de new age travellers en Grande Bretagne », in Cahiers internationaux de sociologie, vol. CXIII (juillet-décembre), 2002, 261-284.

${ }^{24}$ CASTANET, op. cit.

KYROU A., Techno rebelle : Un siècle de musiques électroniques, Denoël, 2002.

SHAPIRO P. et al. (Éd.), Modulations, une histoire de la musique électronique, Paris, Allia, 2004 (1re édition en anglais : 2000). 
Jean-Marie SECA, « Formatage du ressentir et représentations underground », Sociétés, vol. 90, n 4, octobredécembre, 2005, pp. 57-69.

musiciens (crews, tribes, posses) accompagnant un groupe ou un style, il suffit de citer les Merry Pranskters et leur entourage délirant de la fin des années 1960 pour mesurer l'ancienneté du phénomène ${ }^{25}$, sans parler des expériences psychédéliques adoptées en masse en Europe, vingt à trente années après les spéculations et les pratiques oiseuses de l'Américain Timothy Leary et de ses disciples ${ }^{26}$. Déjà à cette époque, un mépris persiflant était formulé à l'encontre des foules adulant les Beatles. N'oublions pas que les « garage bands », ancêtres des groupes punks, se sont développés, en opposition au vedettariat rock et en tant que micro-mouvements provocateurs de consommateurs mécontents. Là aussi, on retrouve des caractéristiques identiques : œuvres artisanalement produites et confidentiellement diffusées sur des bandes enregistrées, groupes restreints, sortis de l'ombre, esprit de distraction (le fun) sans affichage militant, son « distorse », brouillé, minimaliste et bruitiste, montée des « cristaux de masse » vers la diffusion de leur production, imitations foisonnantes du « concept rebelle » initial et nouvelles fragmentations par rapport à ce qui est compris comme un vedettariat insane. Les railleries des adeptes des free parties à l'encontre des « happies » de la « house music » peuvent être comparées à d'autres du même type dans les courants rock (new wave et punks). La disjonction relative entre le rap et la techno peut équivaloir à celle existant entre les tendances parolières des folkeux et les «fioritures soniques » des courants de synphonic ou de progressiv des années 1970. Bien entendu, des différences demeurent et il ne s'agit pas d'assimiler le méandreux style de certaines formations des années 1970 à la trépidante et tonique techno de ce début de siècle. D'un strict point de vue structural, on repère cependant une série de compulsions identiques et une reproduction de quelques attitudes esthétiques entre les anciens styles et les productions contemporaines.

La représentation de la créativité est, en grande partie, commune à divers courants depuis quelques dizaines d'années. Les principaux genres en vogue (rock, rap, techno) et leurs variantes commerciales ou acides, nés entre 1975 et 1985 (reggae, cyberpunk, hip hop, rock new wave et industriel), illustrent, en effet, l'idée d'une pratique autoformatrice et d'une robinsonnade électronique. Bien que les techniques instrumentales aient été l'objet de transformations tout au long de la période 1945-2000, ce qui anime nombre des artisans de ces milieux, est le sentiment de pouvoir réaliser, à partir de «bouts de ficelles » et de moyens réduits, le «futur standard », LE «classique », même et surtout, « underground ». Le grand avantage de cette idée reçue, véhiculée y compris par l'Internet, pour ce qui concerne la techno, est la conviction artisanale. On pense, avec plus de volonté et de consistance qu'auparavant, pouvoir passer en boucle de la création à sa diffusion. II ne s'agit pas d'attendre que les

\footnotetext{
${ }^{25}$ WOLFE T., Acid test, Paris, Seuil, 1975 (1e éd. américaine: 1968).

26 LEARY T., Politique de l'extase, Paris, Fayard, 1973, (1re éd. en langue anglaise : 1968).

LEARY T., Chaos et cyberculture, Paris Le Lézard, 1996 (1e éd. en langue anglaise : 1994).
} 
Jean-Marie SECA, « Formatage du ressentir et représentations underground », Sociétés, vol. 90, $\mathbf{n}^{\circ}$ 4, octobredécembre, 2005, pp. 57-69.

majors et les distributeurs approuvent et décident de répercuter l'enchantement repéré. Les punks, et quelques autres, avant eux, avaient exploré ce chemin sans parler de la légende maudite du Velvet Underground.

\section{Emprises soniques, corps « en transe » et besoins de reconnaissance sociale}

On doit insister sur la recherche de puissance quand on parle de ces pratiques, en apparence, et aussi en réalité, si ludiques. Dans tous les cas, on a affaire à des entités qui fabriquent et font se diffuser des systèmes portatifs d'emprise électro-acoustique. Bien entendu, des différences et des évolutions se font jour depuis le moment punk de 1976 et les « garage bands » des années 1960. J'ai proposé un modèle d'analyse, qualifié d' «état acide », en 1987-1988 et en 2001, qui peut servir à décrire les modes de production de ces musiques. Par un pur hasard, à moins que je n'aie été, sans m'en rendre compte, marqué par l'esprit de ces années, le mot « acide » est réapparu, dans la presse et chez certains groupes, entre 1986 et 1988 . Je ne vais pas tenter de défendre ou de rappeler longuement le contenu de cette grille27. II suffit de dire qu'il s'agit d'une formalisation théorique des pratiques de reconnaissance sociale et de socialisation des ensembles artistiques marginaux sectaires ${ }^{28}$ ou minoritaires. Ce modèle décrit les interrelations entre cinq dimensions :

1. Des ensembles de pratiques artistiques, le plus souvent musicales, formant des systèmes néorituels ;

2. Des représentations connexes, stylistiquement et commercialement marquées ;

3. Un statut spécifique d'acteur créateur ou de praticien (faible reconnaissance et frustration ou exaltation du soi minoritaire) ;

4. Un entrecroisement entre des pratiques (création / diffusion par les répétitions, la production de disques, les concerts, les webzines) et des représentations (« fluide » musical alimentant les rituels, accentuant les effets de sens et la portée des actes de communication sur les destinataires ; textes revendicatifs ou contestataires, quand ils existent) ;

\section{Une recherche plus ou moins avouées et initiatique d'états spéciaux de la conscience et du corps (thème transculturel faisant lien entre les quatre dimensions ci-dessous).}

C'est dans l'hybridation de ces cinq critères que l'on peut voir émerger une Weltanschauung, donc un ensemble de représentations formant un agrégat d'opinions et de pratiques qui se « magnétisent » les

\footnotetext{
27 Seca, op. cit.

${ }^{28}$ ROUQUETTE M.-L., « Eléments pour une théorie minimale des sectes », in Psychologie et société, 3, 2, $2003,9-22$.
} 
Jean-Marie SECA, « Formatage du ressentir et représentations underground », Sociétés, vol. 90, n 4, octobredécembre, 2005, pp. 57-69.

unes les autres et s'ajustent de façon cohérente au fur et à mesure que le monde social s'en rapproche et les incarne. Cette idée d'accentuation de l'emprise d'un tel modèle de socialisation ne pourrait-elle pas constituer une clé d'explication du relatif succès des courants électroniques récents mais aussi des formes rock extrêmes ${ }^{29}$ qui figurent une autre face de l'émergence underground?

Je soutiens, en conséquence, la thèse d'un basculement actuel vers l'underground de masse. Les musiques anticipent, d'abord de façon confidentielle, une intuition des modes d'organisation et de régulation économiques et culturelles ${ }^{30}$. Les premières formes de rock contestataire ou de musique populaire subversive avaient quelque chose de messianique. Elles annonçaient cette nouveauté à venir. Aujourd'hui, le passage du « minoritaire » au «majoritaire » concernant ces styles semble se dérouler sous nos yeux. II se caractérise par l'accroissement des groupements et genres se réclamant d'une attitude underground, innovante ou contemporaine. Beaucoup de praticiens actuels sont des continuateurs de parents amateurs de musique punk ou de grands-parents hippies. Les jeunes générations récentes ont évolué dans une atmosphère d'adoration cultivée de certaines formes « avantgardistes ». Avec ce background éducatif et générationnel, beaucoup ont été « préparées » à mieux s'insérer dans un mode rythmique d'être au monde. Une philosophie clandestine ${ }^{31}$ diablement efficace, de ces styles circule depuis des dizaines d'années. Ceux-ci ont acquis leur « lettres de noblesse », de haute volée, notamment contre des genres institués. Le corporéisme musical et culturel qui accompagne leur emprise psychique n'est pas récent. Jean Maisonneuve et Marilou Bruchon-Scweitzer l'ont clairement décrit, vers la fin des années 1970, dans ses manifestations diverses, tant sur le plan de la vie quotidienne qu'artistique : «Aussi bien dans ces nouvelles pratiques collectives que dans les arts et le théâtre contemporains et jusque dans un certain vécu et un certain discours quotidien, partout on paraît s'orienter électivement vers des émotions et des images irruptives ou fusionnelles ; vers un retour à une Mère-nature (bafouée mais compatissante) tendant à exclure toute loi qui rappellerait celle du père terrible... Finalement, le corporéisme récuse les idéologies normatives d'hier pour leur substituer une sorte de projet vital consistant sinon cohérent, en combinant la restauration narcissique, le désir de tendresse et l'attrait même de la transgression 32 ».

\footnotetext{
29 HEIN, op. cit. ;

LESCOP G., « Honnie soit la oï ! : naissance, émergence et déliquescence d'une forme de protestation sociale et musicale », in Copyright Volume !, vol. 2, n 1, 2003, 109-128.
}

MOMBELET, op. cit. ;

WALZER, op. cit.

30 ATTALI J., Bruits. Essai sur l'économie politique de la musique, Paris, PUF, 1977.

31 MARCUS G., Lipstick Traces. Une histoire secrète du vingtième siècle, Paris, Allia, 1998.

32 MAISONNEUVE J. et BRUCHON-SCHWEITZER M., Modèles du corps et psychologie esthétique, Paris, PUF, 1981. 
Les idées d'opposition culturelle, d'avant-gardisme et de résistance aux industries du spectacle, associées à des orientations esthétiques fondées sur le bruitisme, l'effraction, le déchirement, l'expression d'un soi profond, l'appréhension d'une nature seconde, dite «plus authentique», «émotionnelle», exprimant une identité véritable, le retour récurrent au «sacré sauvage» de l'effervescence enrichissent un terreau propice à la généralisation de ces courants. Leur cachet marginal donne l'impression qu'il s'agit de phénomènes nouveaux et secrets. La multiplicité de leurs pratiques les fait cependant passer à un statut plus ancré socialement. Ne voit-on donc pas que les genres et les pratiques fondés sur le principe du «garage bands » et du home studio pullulent ? II y a eu, entre 1950 et 1990, une massification de l'accès aux loisirs ou aux plages ${ }^{33}$. De façon identique aux vacances du grand nombre, il y a une prolifération des moyens de produire du son et des représentations connectées à ce projet. Une série croissante de hordes, franges et de « morceaux de foules » sont impatients d'exprimer un soi longuement ciselé par l'esprit des temps pop passés, les stylisations corporéisées et les mises en rythme numérisées. Le plus étonnant est que cela marche aussi bien et que l'illusion aimante encore comme si les masses consommatrices et créatives se jetaient avec un élan conformiste presque instinctif dans la même histoire, en parcourant le rite d'une contre-culture flamboyante.

\section{Une standardisation du ressentir}

Le premier rôle de l'anthropologue est d'observer, si possible de façon impliquée, les représentations et les cultures, d'en rendre compte et de proposer, par diverses méthodes, des théories inductives et compréhensives. J'ai tenté, pour ma part, avec mes moyens techniques et théoriques, de m'y employer de mon mieux. II faut cependant en appeler au rôle critique de ce professionnel quand on considère ces activités si percutantes émotionnellement. Cette efflorescence cumulative de styles, de comportements et d'arts « électro-rock » semble fortement fondée sur l'expression, la régulation et la sublimation d'un stress fondamental face aux organisations. Ne conduirait-elle pas à recréer ailleurs, en mieux, dans les fêtes et concerts, et à laisser dans la vacuité, le lundi (s'il n'y a pas de « before » prolongé), un malaise encore plus grand que le mal qu'ils sont censés, dans un premier temps, expurger cathartiquement? En s'imposant sur la scène sociale, même de façon interstitielle, ces formes acides, provocatrices et puissantes n'agrandissent-elles pas la plaie qu'elles se donnent pour but de réduire ? Certes, on peut épiloguer sur le relatif impact apaisant des stylisations artistiques de la rage et de la violence. Diverses interviews confirment l'existence de cet effet. Les adeptes assidus de ces musiques n'ont certainement pas choisi de vivre dans ce monde qui, d'une certaine manière, impose ses rythmes encore plus

\footnotetext{
33 URBAIN J.-D., Les vacances, Paris, Le Cavalier Bleu, 2002.
} 
Jean-Marie SECA, « Formatage du ressentir et représentations underground », Sociétés, vol. 90, n 4, octobredécembre, 2005, pp. 57-69.

inhumains et ses dysharmonies inaudibles. Doit-on, pour autant, s'accommoder de l'intériorisation croissante de ces modes expressifs? Les mouvements révolutionnaires passés promulguaient régulièrement de périodes d'exception (Terreur, Guerres civiles) dans l'intention d'aménager les avenues conduisant à l'avenir radieux démocratique ou socialiste. Les formes rock et électroniques en se diffusant, font aussi disparaître, sans mauvaises intentions, une certaine sensibilité sous les manteaux neigeux de la philosophie d'avant-garde, au nom d'un combat présenté comme noble : celui de l'avenir radieux par la créativité / célébrité pour tous : le fameux «la vie est une œuvre d'art » susurré par Michel Foucault il y a des années. Certes, la force de quelques génies peut aussi s'exercer dans la techno, le rap ou le rock. Tout le monde sait qu'elle ne s'y limite pas. Par leur généralisation, ces styles restreignent les aptitudes de «vision» et d' «audition». Ils formatent des générations toujours plus nombreuses à des interactions fusionnelles. Ils transforment souvent les besoins de sociabilité en un défoulement. Ils essaient de rétablir, de façon hallucinée, un lien perdu. La famille, la tradition ou le rapport individualisé à la communauté, sont altérés profondément par les contraintes du travail, de l'entreprise et du morcellement moderne. Peuvent-ils être « remplacés » ou substitués par ces «étreintes » électro-acoustiques, fondées sur une mise entre parenthèse du monde et de sa diversité ? Ceux qui fréquentent ces fêtes se retrempent dans des états de dépendance psychique de l'homme-masse tout en prenant une distance cynique avec leurs sensations. De plus, cette « fusionnalité » ne dirait pas son nom puisqu'elle n'aboutirait pas à une réelle communion tout recréant paradoxalement du lien social ${ }^{34}$. Cette énergie primitive des mouvements sociaux n'est pas dangereuse en soi. Elle n'a pas attendu le rock et les courants techno pour être instituée, quelles que soient les époques et dans diverses sociétés ${ }^{35}$. Ces espaces festifs accroissent cependant le besoin d'une vie virtuelle. Ils demeurent, indépendamment des substances effectivement toxiques qui s'y consomment, travaillés par un manque, une compulsion à l'addiction marquante et non chimiquement détectable. Ils invitent à l'oubli et à la fuite, le temps de quelques soirées en incitant à revenir pour, encore, ne plus se souvenir.

Même s'ils vivotent sur cette rente de commerce, il ne s'agit pas de rediaboliser les producteurs et les médiateurs de ces styles. Ils semblent plus aptes, de nos jours, à rassembler des individus positivement disposés à se rencontrer dans des circonstances hypersoniques. Ces tendances, qu'on apprécie ou non le terrible volume sonore qu'elles distillent, ont reçu suffisamment de critiques injustifiées de mouvements de parents obsessionnels. Ce qui pourrait cependant demeurer malsain dans cette standardisation et cette mondialisation du ressentir, c'est la fermeture du champ des feelings sur un état

\footnotetext{
${ }^{34}$ MABILON-BONFILS, op.cit.

${ }_{35}$ MOSCOVICI S., La machine à faire des dieux. Sociologie et psychologie, Paris, Fayard, 1988.
} 
Jean-Marie SECA, « Formatage du ressentir et représentations underground », Sociétés, vol. 90, $\mathbf{n}^{\circ}$ 4, octobredécembre, 2005, pp. 57-69.

cataleptique résurgent, provoqué «pour le bien de l'auditeur » et au nom de la comptine d'un village planétaire dont le scintillement gracieux ne séduit plus ${ }^{36}$. L'eldorado de sociétés traditionnelles idylliques est évidemment une vue de l'esprit. Mais on peut pointer une retombée de cet accroissement structuré et général de ce type de ressentir : sommes-nous en train de perdre, avec le concours des « bombardements massifs » des musiques bien plus commerciales, ce que nous avons mis des siècles à gagner quasi naturellement, du fait du mauvais état des routes et de l'incapacité de nos ancêtres à découvrir plus tôt l'usage domestique du pétrole, des ondes hertziennes et de l'électron ? Je veux parler de la diversité foisonnante des sensibilités, des sociétés et de leur anthropologie. C'est dans ce sens, d'enrichissement interculturel, que l'on peut regretter qu'une sorte de pensée unique s'installe sous les apparences les plus anodines. Elle serait animée par des intentions lénifiantes, intellectualistes et distinctives. Elle s'insinuerait avec le souci récréatif des fins de semaine, un peu comme chez ces Occidentaux sincères qui tentent d'aller à la rencontre « authentique » des Touaregs avec leur puissant quatre-quatre. Cette perversion consisterait, le plus fréquemment, dans l'ânonnement d'un vague alphabet contre-culturel. Sa récitation dansée et assourdissante se ferait sans l'existence systématique des repères historiques et des savoirs pluriels de ceux qui ont voulu doctement cracher sur les tombes des Anciens. Alors, évidemment, on peut se cantonner à comptabiliser le nombre de bénévoles dans les soirées gothic ou techno et s'extasier face à l'aptitude mobilisatrice observée. Mais est-ce un argument suffisant face à cette normativité émotionnelle qui s'installe dans les sous-sols en prenant les voies de traverses de la mélancolie et de la marginalité théâtralisée ? Le seul espoir oxygénant, dans un tel contexte, est dans l'essor croissant de produits hybrides mélangeant rap, techno, rock, jazz, «ethnocultures », approches contemporaines ou plus savantes. Ces nouvelles combinaisons pourraient-elles faire sortir les hordes de leurs cavernes esthétiques et les aider à dissoudre ce réflexe de foule et du « restez groupés ! » qui les constitue ?

Le plus important, dans l'affaire, est le quotidien : tous ces courants prétendent se limiter au fun et à la distraction et finissent cependant par happer toute la place vitale et tout le temps libre de leurs praticiens. II faut regarder en face cette tendance totalisante, assujettissante, obsédante. Elle organise un bien-être de remplacement, fondé sur la virtualité de l'art pour l'art. Elle élime les défauts présents dans les organisations et dans la cité. Elle demeure finalement l'élément le plus troublant parce qu'envoûtant.

\footnotetext{
${ }^{36}$ BRETON P., L'utopie de la communication. L'émergence de l'homme sans intérieur, Paris, 1992. WOLTON D., L'autre mondialisation, Paris, Flammarion, 2003.
} 